PROCEEDINGS OF THE AMERICAN MATHEMATICAL SOCIETY

Volume 124, Number 7, July 1996

\title{
DUALITY AND PERFECT PROBABILITY SPACES
}

\author{
D. RAMACHANDRAN AND L. RÜSCHENDORF
}

(Communicated by Richard T. Durrett)

\begin{abstract}
Given probability spaces $\left(X_{i}, \mathcal{A}_{i}, P_{i}\right), i=1,2$, let $\mathcal{M}\left(P_{1}, P_{2}\right)$ denote the set of all probabilities on the product space with marginals $P_{1}$ and $P_{2}$ and let $h$ be a measurable function on $\left(X_{1} \times X_{2}, \mathcal{A}_{1} \otimes \mathcal{A}_{2}\right)$. Continuous versions of linear programming stemming from the works of Monge (1781) and Kantorovich-Rubinštein (1958) for the case of compact metric spaces are concerned with the validity of the duality

$$
\begin{aligned}
& \sup \left\{\int h d P: P \in \mathcal{M}\left(P_{1}, P_{2}\right)\right\} \\
& \quad=\inf \left\{\sum_{i=1}^{2} \int h_{i} d P_{i}: h_{i} \in \mathcal{L}^{1}\left(P_{i}\right) \text { and } h \leq \oplus_{i} h_{i}\right\}
\end{aligned}
$$

(where $\mathcal{M}\left(P_{1}, P_{2}\right)$ is the collection of all probability measures on $\left(X_{1} \times X_{2}, \mathcal{A}_{1} \otimes\right.$ $\mathcal{A}_{2}$ ) with $P_{1}$ and $P_{2}$ as the marginals). A recently established general duality theorem asserts the validity of the above duality whenever at least one of the marginals is a perfect probability space. We pursue the converse direction to examine the interplay between the notions of duality and perfectness and obtain a new characterization of perfect probability spaces.
\end{abstract}

\section{INTRODUCTION}

Let $(X, \mathcal{A}, P)$ be a probability space. $P$ is called perfect (equivalently, the space $(X, \mathcal{A}, P)$ is called perfect) if, for every $\mathcal{A}$-measurable, real-valued function $f$ on $X$ we can find a Borel subset $B_{f}$ of the real line such that $B_{f} \subset f(X)$ with $P\left(f^{-1}\left(B_{f}\right)\right)$ $=1$. Introduced by Gnedenko and Kolmogorov [2] "to achieve complete harmony between the abstract theory of measure and the theory of measures in metric spaces", perfect probability spaces form the technically most pleasing class of probability spaces (see Ramachandran [10]).

A succinct history of the notion of duality from its origins in the works of Monge [5] and Kantorovich-Rubinštein [3], along with a variety of applications in probability theory can be found in Kellerer [4] (see also Dudley [1] and Rachev [9]). Ramachandran and Rüschendorf [11] have recently established that a general duality theorem holds whenever one of the underlying spaces is perfect.

Received by the editors December 15, 1994.

1991 Mathematics Subject Classification. Primary 60A10, 28A35.

Key words and phrases. Duality theorem, marginals, perfect measure, Marczewski function.

Research supported in part by an Internal Awards Grant from the California State University, Sacramento.

(C)1996 American Mathematical Society 
In this note we investigate the converse direction and obtain a new characterization of perfect probability spaces using the notion of duality which brings to light the interplay between the notions of duality and perfectness.

\section{Notation AND PRELIMINARIES}

We use customary measure-theoretic terminology and notation (as, for instance, in Neveu [6]). All measures that we consider are probabilities. For properties of perfect measures we refer the reader to Ramachandran [10]. We denote by $\mathcal{B}$ the $\sigma$-algebra of Borel subsets of $[0,1]$ and by $\lambda$ the Lebesgue measure on $([0,1], \mathcal{B}) . \Delta$ denotes the diagonal in $[0,1] \times[0,1]$. If $P$ is a probability on $(X, \mathcal{A})$, then $P_{*}$ and $P^{*}$ denote respectively the inner and the outer measures induced by $P$. A $\sigma$-algebra $\mathcal{A}_{0}$ is said to be countably generated (or c.g. for short) if $\mathcal{A}_{0}=\sigma\left(\left\{A_{n}, n \geq 1\right\}\right.$ ), in which case $\varphi:\left(X, \mathcal{A}_{0}\right) \rightarrow([0,1], \mathcal{B})$ defined by $\varphi(x)=\sum_{n=1}^{\infty}\left(2 / 3^{n}\right) 1_{A_{n}}(x)$ is called the Marczewski function; $\varphi$ is measurable with $\varphi\left(x_{1}\right) \neq \varphi\left(x_{2}\right)$ if $x_{1}$ and $x_{2}$ belong to different atoms of $\mathcal{A}_{0}$, and so we can identify $\left(X, \mathcal{A}_{0}\right)$ with $(\varphi(X), \mathcal{B} \cap \varphi(X))$. We say that $(X, \mathcal{A}, P)$ is a thick subspace of $\left(X_{1}, \mathcal{A}_{1}, P_{1}\right)$ and write $(X, \mathcal{A}, P) \subset$ $\left(X_{1}, \mathcal{A}_{1}, P_{1}\right)$ whenever $X \subset X_{1}, \mathcal{A}=\mathcal{A}_{1} \cap X=$ the trace of $\mathcal{A}_{1}$ on $X, P_{1}^{*}(X)=1$ and $P=P_{1}^{*} \mid \mathcal{A}$.

Let $\left(X_{i}, \mathcal{A}_{i}, P_{i}\right), i=1,2$, be two probability spaces. A probability $\mu$ on $\left(X_{1} \times\right.$ $\left.X_{2}, \mathcal{A}_{1} \otimes \mathcal{A}_{2}\right)$ is said to have marginals $P_{1}$ and $P_{2}$ if

$$
\begin{aligned}
& \mu\left(A_{1} \times X_{2}\right)=P_{1}\left(A_{1}\right) \text { for all } A_{1} \in \mathcal{A}_{1}, \text { and } \\
& \mu\left(X_{1} \times A_{2}\right)=P_{2}\left(A_{2}\right) \text { for all } A_{2} \in \mathcal{A}_{2} .
\end{aligned}
$$

Let $\mathcal{M}\left(P_{1}, P_{2}\right)=\left\{\mu\right.$ on $\mathcal{A}_{1} \otimes \mathcal{A}_{2}: \mu$ has marginals $P_{1}$ and $\left.P_{2}\right\} . \pi_{i}: X_{1} \times X_{2} \rightarrow$ $X_{i}$ denote the canonical projections for $i=1,2$. The abbreviation $\oplus g_{i}$ is used for $\sum_{i=1}^{2} g_{i} \circ \pi_{i}$.

For a bounded, $\mathcal{A}_{1} \otimes \mathcal{A}_{2}$-measurable function $h$ on $X_{1} \times X_{2}$, the marginal problem is concerned with

$$
S(h)=\sup \left\{\int_{X_{1} \times X_{2}} h d \mu: \mu \in \mathcal{M}\left(P_{1}, P_{2}\right)\right\}
$$

while the dual problem deals with

$$
I(h)=\inf \left\{\sum_{i=1}^{2} \int_{X_{i}} h_{i} d P_{i}: h_{i} \in \mathcal{L}^{1}\left(P_{i}\right) \text { and } h \leq \oplus_{i} h_{i}\right\} .
$$

The measure-theoretic version of the transportation problem dating back to Monge [5] seeks the validity of the duality

$$
S(h)=I(h) .
$$

The main duality theorem of Kellerer [4] deals essentially with second countable or metrizable spaces $X_{i}, i=1,2$, with tight (or Radon) probabilities defined on the Borel sets in which case (D) is shown to hold for a suitably large class containing all the bounded, measurable functions. The following result of Ramachandran and Rüschendorf [11] is the most general duality theorem of this type.

Theorem 1. If at least one of the underlying probability spaces is perfect, then (D) holds for all bounded, measurable functions.

We pursue the converse direction in the next section. 


\section{MAin RESUlts}

We first construct an example to show that the direct converse of Theorem 1 does not hold; that is, (D) can hold for all bounded measurable functions while both the underlying probability spaces are nonperfect.

Example 1. Pachl [8] has constructed a subset $X$ of $[0,1]$ such that

(i) $\lambda^{*}(X)=1=\lambda^{*}([0,1]-X)$ and

(ii) if $\bar{\mu}$ is a probability on $([0,1] \times[0,1], \mathcal{B} \otimes \mathcal{B})$ with $\bar{\mu} \in \mathcal{M}(\lambda, \lambda)$, then $\bar{\mu}^{*}(X \times X)$ $=1$.

Let $X_{i}=X, \mathcal{A}_{i}=\mathcal{B} \cap X$ and $P_{i}=\lambda^{*} \mid \mathcal{A}_{i}$ for $i=1,2$. Then $P_{i}$ is clearly nonperfect for both $i=1,2$. For $\mu \in \mathcal{M}\left(P_{1}, P_{2}\right)$ let $\bar{\mu}$ be defined by $\bar{\mu}(C)=$ $\mu(C \cap(X \times X)), C \in \mathcal{B} \otimes \mathcal{B}$. Then it is easy to check that $\bar{\mu} \in \mathcal{M}(\lambda, \lambda)$ and that $\mu \rightarrow \bar{\mu}$ is a 1-1 correspondence between $\mathcal{M}\left(P_{1}, P_{2}\right)$ and $\mathcal{M}(\lambda, \lambda)$. Further, since $\mathcal{A}_{1} \otimes \mathcal{A}_{2}=\mathcal{B} \otimes \mathcal{B} \cap(X \times X)$, it can be checked (starting with $1_{C}, C \in \mathcal{A}_{1} \otimes \mathcal{A}_{2}$ and using standard measure-theoretic arguments) that for every $h$ on $X_{1} \times X_{2}$ which is $\mathcal{A}_{1} \otimes \mathcal{A}_{2}$ - measurable there is $\bar{h}$ on $[0,1] \times[0,1]$ which is $\mathcal{B} \otimes \mathcal{B}$ - measurable such that $\left.\bar{h}\right|_{X_{1} \times X_{2}}=h$ and $\int h d \mu=\int \bar{h} d \bar{\mu}$. Hence it follows that for every bounded, $\mathcal{A}_{1} \otimes \mathcal{A}_{2}$-measurable $h$,

$$
\begin{aligned}
S(h) & =\sup \left\{\int h d \mu: \mu \in \mathcal{M}\left(P_{1}, P_{2}\right)\right\} \\
& =\sup \left\{\int \bar{h} d \bar{\mu}: \bar{\mu} \in \mathcal{M}(\lambda, \lambda)\right\} \\
& =S(\bar{h}) \\
& =I(\bar{h}) \quad \text { by Theorem } 1 \\
& \geq I(h) \quad \text { by the definition of } I(h)) \\
& \geq S(h) \quad \text { (by the definitions of } I(h) \text { and } S(h))
\end{aligned}
$$

whereby (D) holds.

In order to bring out the interplay between the notions of perfectness and duality we introduce

Definition 1. A probability space $\left(X_{1}, \mathcal{A}_{1}, P_{1}\right)$ is said to be a duality space if for every $\left(X_{2}, \mathcal{A}_{2}, P_{2}\right)$ the duality (D) holds for all bounded, measurable functions.

Theorem 1 can now be recast as

Theorem 2. Every perfect probability space is a duality space.

The next major step is to establish

Proposition 1. If $(X, \mathcal{A}, P)$ is nonperfect, then there exists $\left(X_{1}, \mathcal{A}_{1}, P_{1}\right)$ such that

(i) $(X, \mathcal{A}, P) \subset\left(X_{1}, \mathcal{A}_{1}, P_{1}\right)$ and

(ii) $\left(X_{1}, \mathcal{A}_{1}, P_{1}\right)$ is not a duality space.

Proof. Since $(X, \mathcal{A}, P)$ is nonperfect, there exists a c.g. sub- $\sigma$-algebra $\mathcal{A}_{0}$ of $\mathcal{A}$ such that $P_{0}=P \mid \mathcal{A}_{0}$ is nonperfect (see $P 3$ of Ramachandran [10, p. 26]). Let $\varphi$ be the Marczewski function on $\left(X, \mathcal{A}_{0}\right)$ and let $Q_{0}=P_{0} \varphi^{-1}$. Then $\varphi$ provides the Marczewski identification of the space $\left(X, \mathcal{A}_{0}, P_{0}\right)$ with $\left(\varphi(X), \mathcal{B} \cap \varphi(X), Q_{0}\right) \subset$ $\left([0,1], \mathcal{B}, \bar{Q}_{0}\right)$ where $\bar{Q}_{0}$ is defined on $\mathcal{B}$ by $\bar{Q}_{0}(B)=Q_{0}(B \cap \varphi(X))\left(=P_{0} \varphi^{-1}(B)\right)$, $B \in \mathcal{B}$. Notice that, by construction, $\bar{Q}_{0}^{*}(\varphi(X))=1$.

Suppose that $\bar{Q}_{0 *}(\varphi(X))=1$. Then we can find a set $Y \subset \varphi(X), Y \in \mathcal{B}$, with $\overline{Q_{0}}(Y)=1$. Since $Y$ is a Borel subset of $[0,1]$, every probability on $(Y, \mathcal{B} \cap$ $Y$ ) is perfect (see Theorem 2.3.1 of Ramachandran [10]); hence, $\left.Q_{0}\right|_{Y}$ is perfect which in turn would imply that $Q_{0}$ is perfect. However, $Q_{0}$ is nonperfect since 
$P_{0}$ is nonperfect (see $P 7$ of Ramachandran [10, p. 27]), and so we conclude that $\bar{Q}_{0 *}(\varphi(X))=\alpha<1$.

Let $f: x \longmapsto(x, \varphi(x), \varphi(x)), x \in X$.

Then $f:(X, \mathcal{A}, P) \rightarrow\left(X \times \varphi(X) \times[0,1], \mathcal{A} \otimes(\mathcal{B} \cap \varphi(X)) \otimes \mathcal{B}, P f^{-1}\right)$ has the following properties:

1. $f$ is $1-1$.

2. Since $G_{\varphi}=\{(x, \varphi(x)), x \in X\}=$ Graph of $\varphi \in \mathcal{A} \otimes \mathcal{B} \cap(X \times \varphi(X))=$ $\mathcal{A} \otimes(\mathcal{B} \cap \varphi(X))$, it follows that

$$
f(X)=\left(G_{\varphi} \times[0,1]\right) \cap(X \times(\Delta \cap(\varphi(X) \times[0,1]))) \in \mathcal{A} \otimes(\mathcal{B} \cap \varphi(X)) \otimes \mathcal{B} .
$$

3. $P f^{-1}(f(X))=P(X)=1$.

4. $f^{-1}(A \times(B \cap \varphi(X)) \times C)=A \cap \varphi^{-1}(B) \cap \varphi^{-1}(C) \in \mathcal{A}$ for all $A \in \mathcal{A}, B \in \mathcal{B}$ and $C \in \mathcal{B}$.

5. For $A \in \mathcal{A}, f(A)=(A \times \varphi(X) \times[0,1]) \cap f(X) \in \mathcal{A} \otimes(\mathcal{B} \cap \varphi(X)) \otimes \mathcal{B}$.

Hence, we have

$$
\begin{aligned}
& (X, \mathcal{A}, P) \stackrel{f}{\longleftrightarrow}\left(f(X),(\mathcal{A} \otimes(\mathcal{B} \cap \varphi(X)) \otimes \mathcal{B}) \cap f(X), P f^{-1}\right) \\
& \subset\left(X \times \varphi(X) \times[0,1], \mathcal{A} \otimes(\mathcal{B} \cap \varphi(X)) \otimes \mathcal{B}, P f^{-1}\right) \\
& \stackrel{\text { def }}{=}\left(X_{1}, \mathcal{A}_{1}, P_{1}\right) \text {. }
\end{aligned}
$$

$P_{1}$ has marginals $P, Q_{0}$ and $\bar{Q}_{0}$ respectively on $\mathcal{A}, \mathcal{B} \cap \varphi(X)$ and $\mathcal{B}$ (by 4 above). Define

$$
X_{2}=[0,1] \times(\varphi(X))^{c}, \quad \mathcal{A}_{2}=\mathcal{B} \otimes\left(\mathcal{B} \cap(\varphi(X))^{c}\right) .
$$

Before defining $P_{2}$ observe that $\bar{Q}_{0}^{*}\left((\varphi(X))^{c}\right)=1-\alpha>0$ and $\bar{Q}_{0 *}\left((\varphi(X))^{c}\right)=$ 0 . Choose and fix $B_{0} \in \mathcal{B}, B_{0} \subset \varphi(X)$ such that $\bar{Q}_{0}\left(B_{0}\right)=\bar{Q}_{0 *}(\varphi(X))=\alpha$. Let $Q_{1}$ on $\mathcal{B}$ be defined by

$$
Q_{1}(B)=\bar{Q}_{0}\left(B \mid B_{0}^{c}\right)=\frac{\bar{Q}_{0}\left(B-B_{0}\right)}{1-\alpha}, \quad B \in \mathcal{B} .
$$

Then $Q_{1}\left(B_{0}^{c}\right)=1$ and $B_{0}^{c} \supset(\varphi(X))^{c}$. If $B \in \mathcal{B}$ with $B \supset(\varphi(X))^{c}$, then $B^{c} \subset$ $\varphi(X) \Rightarrow \bar{Q}_{0}\left(B^{c}-B_{0}\right)=0 \Rightarrow Q_{1}\left(B^{c}\right)=0 \Rightarrow Q_{1}(B)=1$ and so $Q_{1}^{*}\left((\varphi(X))^{c}\right)=1$. Similarly, it can be seen that $Q_{1 *}\left((\varphi(X))^{c}\right)=0$. Now define $P_{2}$ on $\mathcal{A}_{2}$ by

$$
P_{2}\left(A_{2}\right)=Q_{1}^{*}\left(\pi_{2}\left(A_{2} \cap \triangle\right)\right), A_{2} \in \mathcal{A}_{2} .
$$

Marginals of $P_{2}$ are $Q_{1}$ and $Q_{1}^{*}$ respectively. Having constructed suitable $\left(X_{i}, \mathcal{A}_{i}\right.$, $\left.P_{i}\right), i=1,2$, with $(X, \mathcal{A}, P) \subset\left(X_{1}, \mathcal{A}_{1}, P_{1}\right)$, we now show that $\left(X_{1}, \mathcal{A}_{1}, P_{1}\right)$ fails to be a duality space.

Consider

$$
X_{1} \times X_{2}=(X \times \varphi(X) \times[0,1]) \times\left([0,1] \times(\varphi(X))^{c}\right)
$$

and define

$$
H=X \times \varphi(X) \times \triangle \times(\varphi(X))^{c} \in \mathcal{A}_{1} \otimes \mathcal{A}_{2} .
$$

Let $h=1_{H}$. Since $P_{1}(X \times(\triangle \cap(\varphi(X) \times[0,1])))=1=P_{2}\left(\triangle \cap X_{2}\right)$, for every $\mu \in \mathcal{M}\left(P_{1}, P_{2}\right)$ we get $\mu\left(X \times(\triangle \cap(\varphi(X) \times[0,1])) \times\left(\triangle \cap X_{2}\right)\right)=1$. But $H \cap(X \times$ $\left.(\triangle \cap(\varphi(X) \times[0,1])) \times\left(\triangle \cap X_{2}\right)\right)=\emptyset$ and so $\mu(H)=0$ for all $\mu \in \mu\left(P_{1}, P_{2}\right)$. Hence $S(h)=0$. 
By (3.3) of Kellerer [4] (see also Strassen [12]), we have

$I(h)=\inf \left\{P_{1}\left(A_{1}\right)+P_{2}\left(A_{2}\right): H \subset\left(A_{1} \times X_{2}\right) \cup\left(X_{1} \times A_{2}\right), A_{i} \in \mathcal{A}_{i}, i=1,2\right\}$.

Since $H \subset\left(\emptyset \times X_{2}\right) \cup\left(X_{1} \times X_{2}\right)$, we get

$$
I(h) \leq P_{1}(\emptyset)+P_{2}\left(X_{2}\right)=1 .
$$

If $H \subset\left(A_{1} \times X_{2}\right) \cup\left(X_{1} \times A_{2}\right)$ with $A_{i} \in \mathcal{A}_{i}, i=1,2$, then let

$$
B_{2}=\pi_{2}\left(A_{2} \cap \triangle\right) \in \mathcal{B} \cap(\varphi(X))^{c} .
$$

Hence, $B_{2}=B \cap(\varphi(X))^{c}$ for some $B \in \mathcal{B}$ and so

$$
P_{2}\left(A_{2}\right)=Q_{1}^{*}\left(B_{2}\right)=Q_{1}(B)=\frac{\bar{Q}_{0}\left(B-B_{0}\right)}{1-\alpha} .
$$

Carefully look at

$$
\begin{aligned}
& D=X \times \varphi(X) \times\left(B^{c} \cap(\varphi(X))^{c}\right) . \\
&\left(x, y_{1}, y_{2}\right) \in D \Rightarrow\left(x, y_{1}, y_{2}, y_{2}, y_{2}\right) \in H \\
& \Rightarrow\left(x, y_{1}, y_{2}, y_{2}, y_{2}\right) \in A_{1} \times X_{2} \\
&\left(\left(y_{2}, y_{2}\right) \notin A_{2} \text { since } y_{2} \notin B_{2}\right) \\
& \Rightarrow\left(x, y_{1}, y_{2}\right) \in A_{1} .
\end{aligned}
$$

It follows that $D \subset A_{1}$. Hence

$$
P_{1}\left(A_{1}\right) \geq P_{1}^{*}(D)=\bar{Q}_{0}^{*}\left(B^{c} \cap(\varphi(X))^{c}\right)=\bar{Q}_{0}\left(B^{c}-B_{0}\right) .
$$

(To conclude that $\bar{Q}_{0}^{*}\left(C \cap(\varphi(X))^{c}\right)=\bar{Q}_{0}\left(C-B_{0}\right)$ for all $C \in \mathcal{B}$ first note that $\bar{Q}_{0}\left(C-B_{0}\right) \geq \bar{Q}_{0}^{*}\left(C \cap(\varphi(X))^{c}\right)$ since $B_{0} \subset \varphi(X)$; on the other hand, if $C \cap(\varphi(X))^{c} \subset C_{1} \in \mathcal{B}$, then $\left(C-B_{0}\right)-\left(C_{1}-B_{0}\right) \subset \varphi(X)-B_{0} \Rightarrow \bar{Q}_{0}\left(\left(C-B_{0}\right)-\right.$ $\left.\left.\left(C_{1}-B_{0}\right)\right)=0 \Rightarrow \bar{Q}_{0}\left(C-B_{0}\right)=\bar{Q}_{0}\left(\left(C-B_{0}\right) \cap\left(C_{1}-B_{0}\right)\right) \leq \bar{Q}_{0}\left(C_{1}-B_{0}\right) \leq \bar{Q}_{0}\left(C_{1}\right).\right)$

Adding (1) and (2),

$$
\begin{aligned}
P_{1}\left(A_{1}\right)+P_{2}\left(A_{2}\right) & \geq \frac{\bar{Q}_{0}\left(B-B_{0}\right)}{1-\alpha}+\bar{Q}_{0}\left(B^{c}-B_{0}\right) \\
& \geq \bar{Q}_{0}\left(B-B_{0}\right)+\bar{Q}_{0}\left(B^{c}-B_{0}\right) \\
& =\bar{Q}_{0}\left(B_{0}^{c}\right)=1-\alpha .
\end{aligned}
$$

Thus $I(h) \geq 1-\alpha>0$ and as a consequence $\left(X_{1}, \mathcal{A}_{1}, P_{1}\right)$ fails to be a duality space. is

Our main result which yields a new characterization of perfectness of measures

Theorem 3. Let $(X, \mathcal{A}, P)$ be a probability space. Then the following statements are equivalent:

(a) $(X, \mathcal{A}, P)$ is perfect.

(b) If $(X, \mathcal{A}, P) \subset\left(X_{1}, \mathcal{A}_{1}, P_{1}\right)$, then $\left(X_{1}, \mathcal{A}_{1}, P_{1}\right)$ is perfect.

(c) If $(X, \mathcal{A}, P) \subset\left(X_{1}, \mathcal{A}_{1}, P_{1}\right)$, then $\left(X_{1}, \mathcal{A}_{1}, P_{1}\right)$ is a duality space.

Proof. (a) $\Rightarrow$ (b). If $f_{1}$ is an $\mathcal{A}_{1}$-measurable, real-valued function on $X_{1}$, then letting $f=\left.f_{1}\right|_{X}$, choose a Borel set $B_{f}$ of the real line such that $B_{f} \subset f(X) \subset$ $f_{1}\left(X_{1}\right)$ with $P\left(f^{-1} B_{f}\right)=1$. Take $B_{f_{1}}=B_{f}$.

(b) $\Rightarrow$ (c) follows from Theorem 2 .

(c) $\Rightarrow$ (a) is Proposition 1. 


\section{REFERENCES}

1. Dudley, R.M.: Probabilities and Metrics. Lecture Notes Series No. 45. Aarhus: Matematisk Institut, 1976. MR 58:7764

2. Gnedenko, B.V., and Kolomogorov, A.N. (1954). Limit distributions for sums of independent random variables. Addison Wesley, Cambridge. MR 16:52d

3. Kantorovich, L.V., and Rubinštein, G.S. (1958). On a space of completely additive functions (in Russian). Vestnik Leningrad Univ . 13/7, 52-59. MR 21:808

4. Kellerer, H.G. (1984). Duality Theorems for marginal problems. Z. Wahrscheinlichkeitstheorie verw. Gebiete 67, 399-432. MR 86i: 28010

5. Monge, G. (1781). Mémoire sur la théorie des déblais ét ramblais. Mem. Math. Phys. Acad. Roy. Sci. Paris, 666-704.

6. Neveu, J. (1965). Mathematical foundations of the calculus of probability. Holden Day, London. MR 33:6660

7. Pachl, J. (1979). Two classes of measures. Colloq. Math. 42, 331-340. MR 82b:28012

8. Pachl, J. (1981). Correction to the paper "Two classes of measures. " Colloq. Math. 45, 331-333. MR 84c:28009

9. Rachev, S.T. : Probability metrics and the stability of stochastic models. New York: Wiley 1991 MR 93b:60012

10. Ramachandran, D. (1979). Perfect Measures, I and II. ISI Lecture Notes Series, 5 and 7 , New Delhi, Macmillan. MR 81h:6005b; MR 81h:6005a

11. Ramachandran, D. and Rüschendorf, L. (1995). A general duality theorem for marginal problems. Probab. Theory Relat. Fields, 101, 311-319.

12. Strassen, V. (1965). The existence of probability measures with given marginals. Ann. Math. Statist. 36, 423-439. MR 31:1693

Department Of Mathematics and Statistics, California State University, $6000 \mathrm{~J}$ Street, Sacramento, California 95819-6051

E-mail address: chandra@csus.edu

California State University, Sacramento and Universität Freiburg

Current address: Institut für Mathematische Stochastik, Albert-Ludwigs-Universität, Hebelstr. 27, D-79104 Freiburg, Germany

E-mail address: ruschen@buffon.mathematik.uni-freiburg.de 